TITLE:

\title{
MOLLUSCS SYMBIOTIC WITH COELENTERATES IN JAPAN, WITH SPECIAL REFERENCE TO OVULIDAE AND ALLIED FORMS
}

\author{
$\operatorname{AUTHOR}(\mathrm{S}):$ \\ Yamamoto, Torao
}

\section{CITATION:}

Yamamoto, Torao. MOLLUSCS SYMBIOTIC WITH COELENTERATES IN JAPAN, WITH SPECIAL REFERENCE TO OVULIDAE AND ALLIED FORMS. PUBLICATIONS OF THE SETO MARINE BIOLOGICAL LABORATORY 1973, 20: 567-581

ISSUE DATE:

1973-12-19

URL:

http://hdl.handle.net/2433/175758

RIGHT: 


\title{
MOLLUSCS SYMBIOTIC WITH COELENTERATES IN JAPAN, WITH SPECIAL REFERENCE TO OVULIDAE AND ALLIED FORMS ${ }^{11}$
}

\author{
TORAO YAMAMOTO \\ Nanki Biological Society, Sirahama, Wakayama Prefecture
}

With 12 Text-figures

The molluscs hitherto recorded as symbiotic with coelenterates in Japanese waters comprize the following groups: Fam. Pteridae (Lamellibranchia) with genera Pteria and Pterelectroma (with gorgonians and hydrocorals), Fam. Ovulidae (Gastropoda) with genera Primovula and Prionovolva (with gorgonians, alcyonarians, and hydrocorals), Fams. Montactidae and Galeommatidae (Lamellibranchia) respectively with genera Nipponomontacuta and Ephippodonta (both with anthozoans), Fams. Pholadidae and Mytilidae (Lamellibranchia) respectively with genera Jouannecta and Lithophaga, Fam. Coralliophilidae (Gastropoda) with five genera comprising popular Coralliophilla (with stony corals), and Fams. Epitoniidae and Architectonicidae (Gastropoda) respectively with genera Gyroscala and Spiniscala and with genus Heliacus (with sea anemones).

These molluscs may then be classified into the following three ecological groups:

1. Merely attaching externally $\ldots \ldots \ldots \ldots \ldots \ldots \ldots \ldots \ldots$. Pteriidae

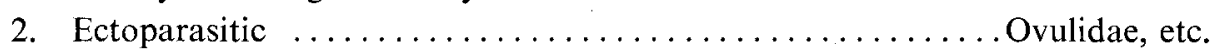

3. Boring into the soft part or skeletal structure of host ..... Coralliophilla,

Lithophaga, etc.

Of these symbiotic molluscs, members of Fam. Ovulidae and allied forms are apparently most remarkable in number of species and seemingly in their adaptive morphology and behaviour. This group is represented in Japanese waters by following 26 species of 7 genera in 2 families, whose hosts have been so far confirmed, though about fifty species have been recorded inclusive of those, of which no records are available to learn their hosts.

Family Ovulidae
1. Primovula striatula (SOWERBY)
5. $P$. myrakeenae Azuma \& CATE
2. P. formosa (ADAMS \& REEVE)
6. $P$. tigris T. YамAMOTO
3. $P$. frumentum (SOWERBY)
7. $P$. tosaensis Azuma \& CATE
4. $P$. rhodia (A. AdAMs)
8. P. (Dentiovula) dorsuosa (HINDS)

1) Contributions from the Seto Marine Biological Laboratory, No. 574. 
9. P. (Prosimnia) coarctata (AdAMs \& REEVE)

10. Prionovolva fruticum (REEVE)

11. $P$.

12. $P$. bulla (ADAMS \& REEvE) hervieri (HEDLEY)

13. $P$. brevis (SOWERBY)

14. Diminovula marginata (SowerBY)

15. $D$. margarita (SOWERBY)

16. $D$.

17. $D$. punctata (Duclos) cristallina (KIENER)

18. D. florida (KurodA)

19. Phenacovolva birostris (LINNÉ)

20. Ph. longirostris (SOWERBY)

21. $P h$ r recurva (AdAMS \& REEVE) REEVE)

22. $P h$. sowerbiana (WEINKAUFF)

23. Ph. (Pellasimnia) subreflexa (Adams \& REEVE)

24. Kuroshiovolva shingoi Azuma \& CATE

25. Ovula ovum LINNÉ

Family Pediculariidae

\section{Pedicularia (Pediculariona) pacifica PEASE}

The writer would like to express his hearty gratitude to Dr. Tokubei KuroDa who has guided him during the course of the present study. Cordial thanks are due to Prof. Huzio Utinomi of the Seto Marine Biological Laboratory for identification of host coelenterates and to Messrs. Kentarô SASAKI and Kinya MASE for valuable specimens and ecological information. The writer is indebted to Messrs. Tadasi TANAKa, Chûichi Araga and Dr. Shin'ichiro FusE for some important photographs appeared in this paper.

\section{Family Ovulidae}

\section{Genus Primovula THIELE}

\section{Primovula striatula (SOWERBY)}

1828 Ovulum striatulum Sowerby, Zool. Jour., 4, p. 155.

1848 Ovulum dentatum Adams \& ReEve, Zool. Samarang, Moll., 21, pl. 6, figs. 4a, b (non FischeR De WaLDHEIM, 1807).

Host and locality confirmed: Gorgonid, Euplexaura erecta KüKENTHAL, (Seto, Sirahama, Wakayama Prefecture, February 1959; Tôsima, Sirahama, Wakayama Prefecture, September 1963; Seto, Sirahama, Wakayama Prefecture, February 1963; Minabe, Wakayama Prefecture, February 1969)

Distribution: Honsyû (northerly to Sagami Bay), Sikoku and Kyûsyû in Japan. Widely in the Indo-Pacific Region. (type locality-Indonesia).

\section{Primovula formosa (ADAMS \& REEVE)}

1848 Ovulum formosum ADAms \& ReEve, Zool. Samarang, Moll., p. 22, pl. 6, fig. 6.

1856 Amphiperas (Ovulum) trailli A. Adams, Proc. Zool. Soc., London, 23 (298), (1855), p. 222.

Host and locality confirmed: Gorgonid, Euplexaura sanguinea UтімомI, (Seto, Sirahama, Wakayama Prefecture, April 1958).

Gorgonid, Euplexaura rigida KüKENTHAL, (Seto, Sirahama, Wakayama Prefecture, March 1959). 
Gorgonid, Euplexaura erecta Kükenthal, (Seto, Sirahama, Wakayama Prefecture, April 1960).

Gorgonid, Echinogorgia praelonga (RIDLEY), (Tôsima, Sirahama, Wakayama prefecture, May 1962).

Distribution: Honsyû (northerly to Bôsô peninsula), Sikoku, Kyûsyû and IndoPacific Region. (type locality-Japan).

\section{Primovula frumentum (SowERBY)}

1828 Ovulum frumentum Sowerby, Zool. Jour., 4 (14), p. 155.

1865 Ovulum frumentum REEVE, Conch. Icon., Ovulum, sp. 25.

Host and locality confirmed: Alcyonarian, Stereonephthya japonica UTINomI, (Bansyozaki, Sirahama, Wakayama Prefecture, August 1952; Tôsima, Sirahama, Wakayama Prefecture, May 1958).

Alcyonarian, Stereonephthya rubriflora UTINomi, (Tôsima, Sirahama, Wakayama Prefecture, June 1958).

Alcyonarian, Dendronephthya gigantea (VERriLl), (Bansyozaki, Sirahama, Wakayama Prefecture, March 1958).

Gorgonid, Euplexaura erecta KüKenthal, (Seto, Sirahama, Wakayama Prefecture, February 1963).

Distribution: Honsyû (northerly to Bôsô peninsula), Sikoku, Kyûsyû. Tropical Pacific region. (type locality unknown).

\section{Primovula rhodia (A. AdAMs)}

1830 Ovulum triticum Sowerby, in Broderip \& SOWERBy, Spec. Conch., Ovulum, 1 (1), p. 6 (non Lamarck, 1810).

1855 Amphiperas rhodia A. Adams, Proc. Soc., London, 22 (1854), p. 130.

Host and locality confirmed: Alcyonarian, Stereonephthya japonica UTINomI, (Bansyozaki, Sirahama, Wakayama Prefecture, May 1958).

Gorgonid, Melithaea flabellifera (KüKenthaL), (Kozima, Ôkawa, Ôsaka Prefecture, September 1959, collected by I. Hamatani; Seto, Sirahama, Wakayama Prefecture, July 1959; Sirasaki, Yura, Wakayama Prefecture, May 1961; Katu'ura, Wakayama Prefecture, June 1966).

Gorgonid, Euplexaura erecta Kükenthal, (Seto, Sirahama, Wakayama Prefecture, August 1960; Bansyozaki, Sirahama, Wakayama Prefecture, August 1960; Tôsima, Sirahama, Wakayama Prefecture, July 1970).

Distribution: Southern Hokkaidô, Honsyû, Sikoku, Kyûsyû, Korea and China. (type locality-Japan).

\section{Primovula myrakeenae Azuma \& CATE}

1970 Primovula myrakeenae Azuma \& CATE, Veliger, 13, p. 263, fig. 7.

Host and locality confirmed: Gorgonid, Acalycigorgia inermis (HEDLUND), 
(Sakai, Minabe, Wakayama Prefecture, November 1971).

Distribution: Honsyû (Sagami Bay and type locality-off Kirimezaki, Wakayama Prefecture).

\section{Primovula tigris T. ҮАмамото}

1971 Primovula tigris T. Yamamoto, Publ. Seto Mar. Biol. Lab., 19 (4), 191-195, pl. 8, figs. 1-3.

Host and distribution confirmed: Gorgonid, Euplexaura erecta KüKENTHAL (off Yuzaki, Sirahama, Wakayama Prefecture, December 1957; off Yuzaki, Sirahama, Wakayama Prefecture, March 1961; Bansyozaki, Sirahama, Wakayama Prefecture, July 1963; Maguti, Miura Peninsula, Kanagawa Prefecture, April 1971, collected by K. MASE).

Distribution: Honsyû (Sagami Bay and type locality-off Yuzaki, Sirahama, Wakayama Prefecture).

\section{Primovula tosaensis Azuma \& Cate}

1971 Primovula tosaensis Azuma \& Cate, Veliger, 13 (3), p. 264-265, figs. 9, 18.

Host and locality confirmed: Gorgonid, Antipathes japonica BrooK, (Sakai, Minabe, Wakayama Prefecture, April 1972, collected by K. SASAKI).

Distribution: Honsyû (northerly to Sagami Bay), Sikoku (type locality-off Kirimezaki, Wakayama Prefecture).

\section{Primovula (Dentiovula) dorsuosa (HINDs)}

1844 Ovulum dorsuosum Hinds, Zool. Sulphur, p. 47, pl. 16, figs. 3, 4.

1930 Prosimnia renovata Iredale, Mem. Queensl. Mus., 10, p. 85.

1932 Primovula dorsuosa, SCHILder, Proc. Malac. Soc. London, 20, pp. 52, 58.

1968 Primovula striatula GeRnOHORSKY, Veliger, 10 (4), p. 361, pl. 50. fig. 8.

Host and locality confirmed: Gorgonid, Acanthogorgia japonica KüKenthal \& Gorzawsky, (Sakai, Minabe, Wakayama Prefecture, December 1971).

Gorgonid, Acalycigorgia grandiflora KüKenthal \& GoRzawsky, (Iti-no-hai, near Uwazima, Ehime Prefecture, August 1964, collected by T. ITO).

Distribution: Honsyû (northerly to Bôsô peninsula), Sikoku and Kyûsyû. Also in the Western Pacific Region. (type locality-Straits of Malacca).

\section{Primovula (Prosimnia) coarctata (Adams \& ReEve)}

(Figs. 1-2)

1848 Ovulum coarctata Adams \& ReEve, Zool. Samarang, Moll., 21, pl. 6, figs. 2 a, b.

1881 Ovulum sempieri WeInKauff, Conch. Cab., ed. 2, 5, 190, pl. 48, figs. 14, 15.

Host and locality confirmed: Gorgonid, Melithaea flabellifera (KüKENTHAL), (Sirasaki, Yura, Wakayama Prefecture, July 1947; Seto, Sirahama, Wakayama Prefecture, September 1963; Tôsima, Sirahama, Wakayama Prefecture, November 1963; Tôsima, Sirahama, Wakayama Prefecture, September 1970; Sihara, Hikigawa, Wakayama Prefecture, October 1972). 
Gorgonid, Melithaea ocracea (LinnÉ), (Mirozu, Susami, Wakayama Prefecture, November 1970 , collected by K. KITAO)

Distribution: Honsyû (northerly to Bôsô peninsula), Sikoku and Kyûsyû, Also in the Indo-Pacific Region. (type locality Straits of Sunda, near Java).

\section{Genus Prionovolva Iredale}

\section{Prionovolva fruticum (REEVE)}

1848 Ovula nubeculatum Adams \& Rfeve, Zool. Samarang, Moll., P. 23, pl. 6, fig. 12.

1865 Ovulum fruticum ReEve, Conch. Icon., Ovulum, sp. 16.

1932 Prionovolva fruticum, SCHILder, Proc. Malac. Soc. London, 20, pp. 53, 59, pl. 4, fig. 35.

Host and locality confirmed: Alcyonarian, Dendronephthya (Mörchellana) habereri Kükenthal, (Ezura, Sirahama, Wakayama Prefecture, April 1956; Setozaki, Sirahama, Wakayama Prefecture, January 1960; Sionomisaki, Wakayama Prefecture, May 1960; Nada, Gobô, Wakayama Prefecture, January 1960; Ezura, Sirahama, Wakayama Prefecture, January 1961 ; Nada, Gobô, Wakayama Prefecture, April 1962; Sionomisaki, Wakayama Prefecture, March 1971).

Alcyonarian, Dendronephthya (Roxasia) golgotha UTinomi, (Nada, Gobô, Wakayama Prefecture, February 1962)

Alcyonarian, Dendronephthya (Dendronephthya) gigantea (VERRILL), (Nada, Gobô, Wakayama Prefecture, March 1971; Sisôzima, Sirahama, Wakayama Prefecture, March 1971; Sionomisaki, Wakayama Prefecture, March 1971).

Alcyonarian, Dendronephthya (Dendronephthya) nipponica UTiNomi, (Sionomisaki, Wakayama Prefecture, April 1972).

Distribution: Honsyû (northerly to Bôsô Peninsula), Sikoku, Kyûsyû and also in the western Pacific Region. (type locality-Malacca).

\section{Prionovolva bulla (ADAMs \& ReEve)}

1848 Ovulum bulla Adams \& ReEve, Zool. Samarang, Moll., p. 21, pl. 6, figs. 5a, b.

1932 Prionovolva bulla, SchiLder, Proc. Malac. Soc. London, 20, pp. 53, 59, pl. 4, figs. 27, 28.

Host and locality confirmed: Alcyonarian, Dendronephthya (Dendronephthya) gigantea (VERriLL), (Sakai, Minabe, Wakayama Prefecture, March 1968, collected by K. SASAKI)

Alcyonarian, Dendronephthya (Roxasia) golgotha UTINomI, (Nada, Gobô, Wakayama Prefecture, May 1965)

Distribution: Honsyû (northerly to Sagami Bay), Sikoku, Kyûsyû, China Seas, Malayan Archipelago from Malacca to New Guinea. (type locality Malacca).

\section{Prionovolva hervieri (HEDLEY)}

1899 Ovula hervieri Hedley, Mem. Austr. Mus., 3, pp. 448, 449.

1930 Prionovolva hervieri, Iredale, Qeensl. Mus., 10, pl. 1, pp. 73-88, pl.9.

1932 Primovula hervieri, Schilder, Proc. Malac. Soc. London, 20, pp. 51, 58. 
Host and locality confirmed: Alcyonarian, Dendronephthya (Roxasia) suensoni (Holm), (Seto, Sirahama, Wakayama Prefecture, April 1954).

Alcyonarian, Dendronephthya (Dendronephthya) nipponica UTINOMI, (Takui, near Gobô, Wakayama Prefecture, December 1963; Seto, Sirahama, Wakayama Prefecture, March 1964; Sionomisaki, Wakayama Prefecture, March 1971; Sionomisaki, Wakayama Prefecture, May 1971).

Alcyonarian, Dendronephthya (Morchellana) habereri Kükenthal, (Seto, Sirahama, Wakayama Prefecture, December 1957; Sionomisaki, Wakayama Prefecture, March 1959; Sionomisaki, Wakayama Prefecture, May 1960; Sionomisaki, Wakayama Prefecture, January 1961; Mirozu, Susami, Wakayama Prefecture, December 1970; Sionomisaki, Wakayama Prefecture, May 1971).

Alcyonarian, Dendronephthya (Dendronephthya) gigantea (VerRILL), (Seto, Sirahama, Wakayama Prefecture, April 1959; Seto, Sirahama, Wakayama Prefecture, November 1959; Sionomisaki, Wakayama Prefecture, April 1957; Sionomisaki, Wakayama Prefecture, May 1960; Sionomisaki, Wakayama Prefecture, January 1961; Ezura, Sirahama, Wakayama Prefecture, April 1961; Kusimoto, Wakayama Prefecture, March 1962; Sionomisaki, Wakayama Prefecture, March 1971; Sionomisaki, Wakayama Prefecture, March 1972).

Distribution: Honsyû (northerly to Sagami Bay), Sikoku, Kyûsyû and Micronesia (type locality-Funafuti, Qeensland).

\section{Prionovolva brevis (SOWERBY)}

1828 Ovulum breve SowerBy, Zool. Jour., 4 (14), pp. 152, 153.

1913 Ovula nipponensis Prlsbry, Nautilus, 26 (10), pp. 114-115, pl. 7, fig. 4.

1932 Prionovolva brevis (Sowerby), Proc. Malac. Soc. London, 20, p. 53, pl. 4, figs. $29-31$.

1971 Prionovolva (Prionovolva) nebula AzUMA \& CATE, Veliger, 13 (3), p. 262, fig. 4.

Host and locality confirmed: Alcyonarian, Dendronephthya (Dendronephthya) gigantea (VerriLL), (Seto, Sirahama, Wakayama Prefecture, April 1959)

Alcyonarian, Dendronephthya (Morchellana) habereri KüKenthal, (Nada, Gobô, Wakayama Prefecture, April 1960; Sionomisaki, Wakayama Prefecture, May 1960) Alcyonarian, Dendronephthya (Morchellana) castanea UTıNomi, (Sisôzima, Sirahama,

Fig. 1. Primovula (Prosimnia) coarctata (Adams \& Reeve), 12mm in length. Tôsima, Sirahama. Wakayama Prefecture, September 1962 (collected by Mr. K. SASAKI).

Fig. 2. Primovula (Prosimnia) coarctata (ADAMs \& ReEve) on the gorgonid, Melitaea flabellifera (Kükenthal), Tôsima, Sirahama, Wakayama Prefecture, September 1963 (photo by Mr. T. TANAKA).

Fig. 3. Diminovula punctata (Duclos), 18mm in length. Tôsima, Sirahama, Wakayama Prefecture, June 1958.

Fig. 4. Egg ribbon of Diminovula punctata laid on the Alcyonarian, Dendronephthya gigantea (VerrILL), Tôsima, Sirahama,Wakayama Prefecture, August 1963 (collected by Mr. K. SaSAKI and photo by Mr. T. TANAKA).

Fig. 5. Diminovula cristallina (KIENER) on the Alcyonarian, Stereonephthya japonica UTINomI, Seto, Sirahama, Wakayama Prefecture, September 1963 (photo by Mr. T. TANAKA). 

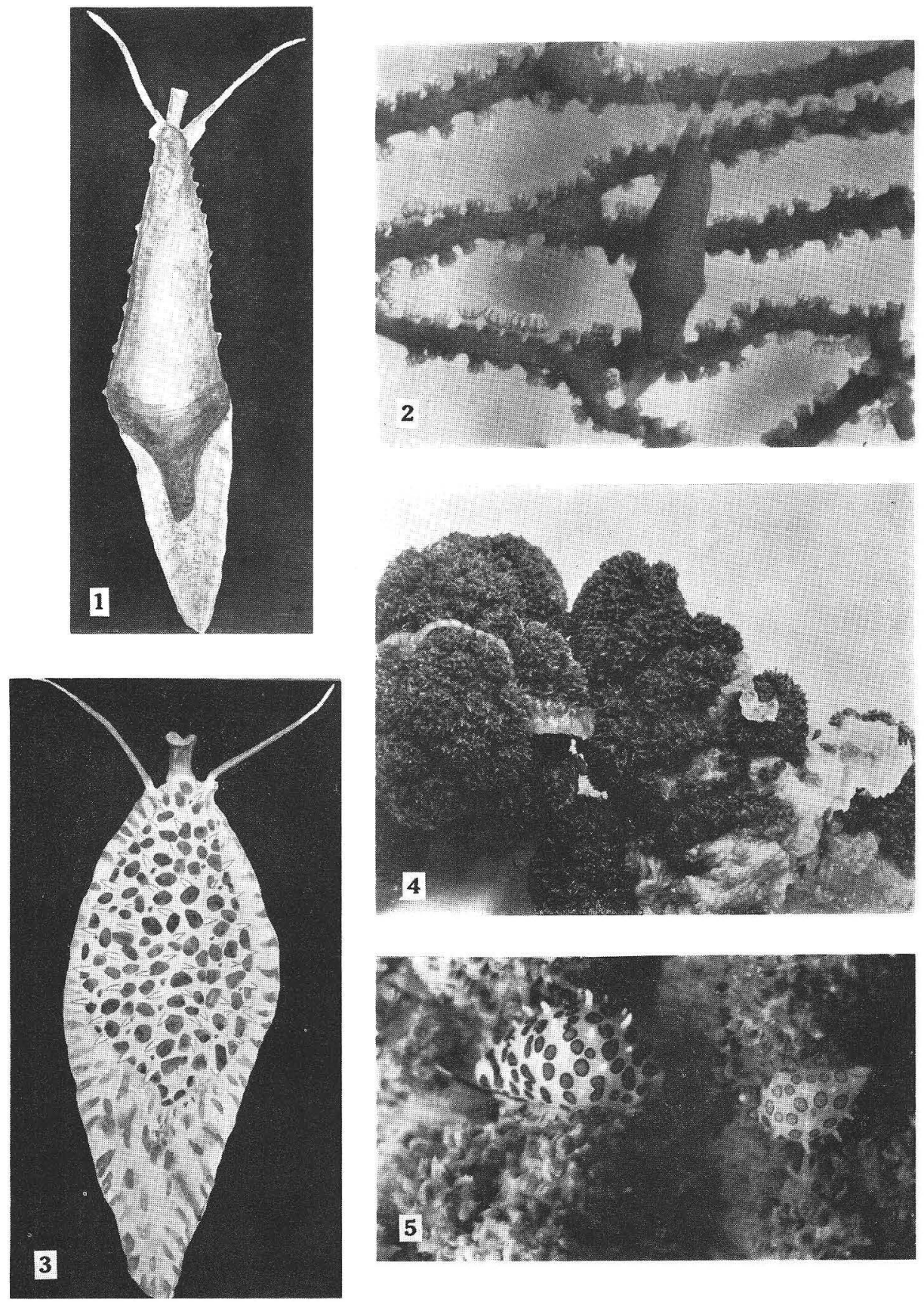

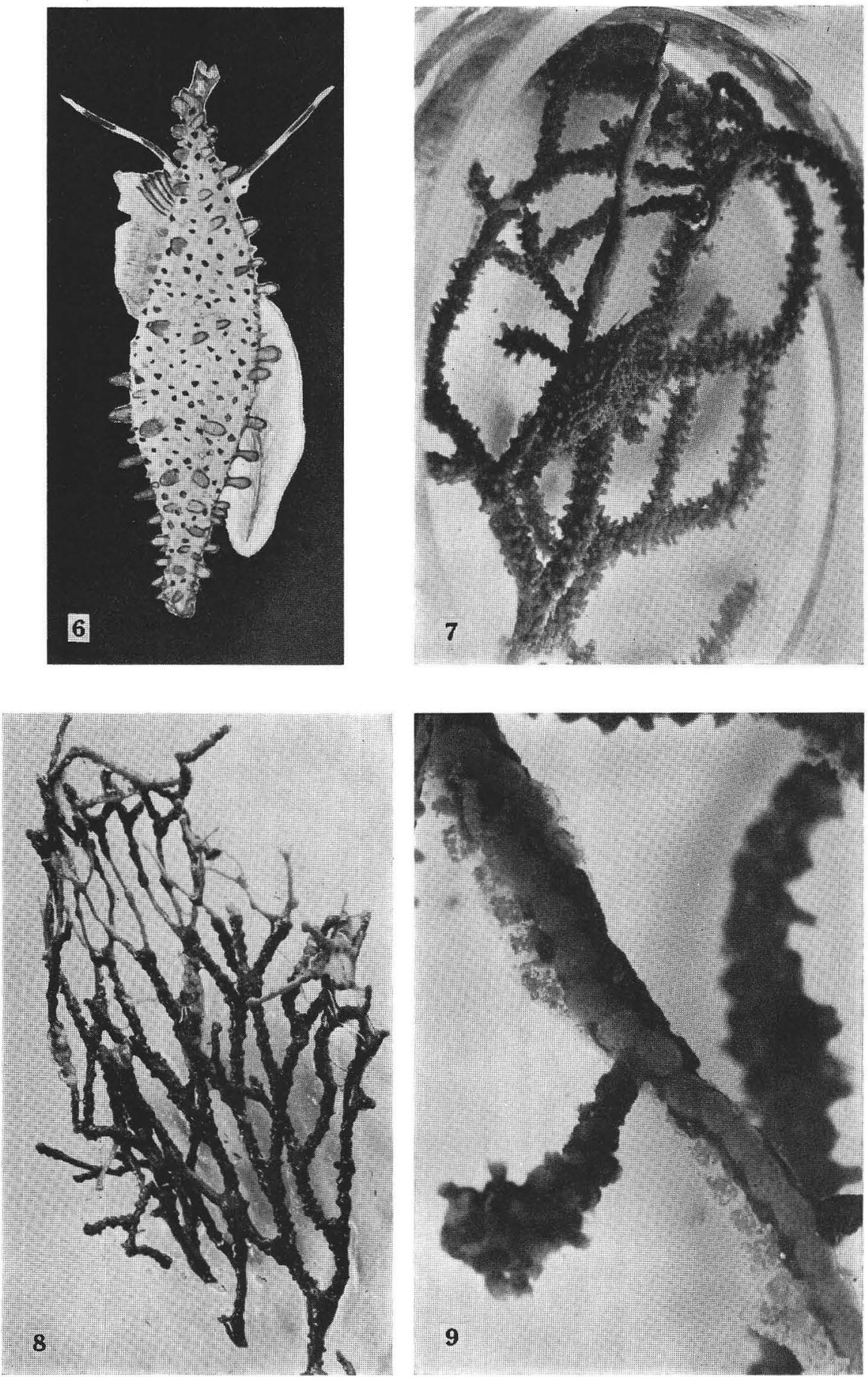
Wakayama Prefecture, March 1971)

Distribution: Honsyû (northerly to Sagami Bay), Sikoku, Kyûsyû and IndoPacific Region. (type locality-Australia)

\section{Genus Diminovula IREDAle}

\section{Diminovula marginata (SOWERBY)}

1828 Ovulum marginata Sowerby, Zool. Jour., 4 (14), p. 151.

1862 Ovulum marginatum ReEve, Conch. Icon., Ovulum, sp. 8.

1932 Prinovolva marginata Schilder, Proc. Malac. Soc. London, 20, pp. 53, 59, pl. 4, figs. 33, 34.

1942 Primovula marginata YeN, Proc. Malac. Soc. London, 24, p. 212, pl. 16, fig. 98.

Host and locality confirmed: Alcyonarian, Dendronephthya tuberculata UTINomI, (Nada, Gobô, Wakayama Prefecture, April 1970, collected by Y. ToKı; Sionomisaki, Wakayama Prefecture, March 1971)

Alcyonarian, Stereonephthya japonica Utinomi (Nagasima, Mie Prefecture, collected by S. SiraI).

Distribution: Honsyû (northerly to Bôsô Peninsula), Sikoku, Kyûsyû, Formosa and China. (type locality-China).

\section{Diminovula margarita (SOWERBY)}

1828 Ovulum margarita Sowerby, Zool. Jour., 4, p. 150.

1849 Ovulum umbilicatum Sowerby, Proc. Zool. Soc. London, 16 (188), p. 135.

1932 Primovula (Diminovula) margarita, Schilder, Proc. Malac. Soc. London, 20, pp. 51, 58, pl. 3, fig. 1.

Host and locality confirmed: Alcyonarian, Stereonephthya rubriflora UTINomi, (Seto, Sirahama, Wakayama Prefecture, July 1953; Seto, Sirahama, Wakayama Prefecture, May 1954; Tôsima, Sirahama, Wakayama Prefecture, June 1958)

Alcyonarian, Stereonephthya japonica UTINomI, (Tôsima, Sirahama, Wakayama Prefecture, June 1958; Bansyozaki, Sirahama, Wakayama Prefecture, May 1959)

Distribution: Honsyû (northerly to Bôsô Peninsula), Sikoku, Kyûsyû, Formosa and China. (type locality-China)

\section{Diminovula punctata (DUClos)}

(Figs. 3-4)

1828 Ovula punctata Duclos, Mem. Soc. Hist. Nat. Paris, 4, p. 248.

Fig. 6. Phenacovolva birostris (LINNÉ), 35mm in length. Tôsima, Sirahama, Wakayama Prefecture, May 1962 (collected by Mr. H. INAGAKI).

Fig. 7. Phenacovolva birostris (LINNÉ) on the gorgonid, Anthoplexaura dimorpha KÜKENTHAL, Tôsima, Sirahama, Wakayama Prefecture, September 1963 (collected by Mr. K. SASAKI and photo by Mr. T. TANAKA).

Fig. 8. Anthoplexaura dimorpha KÜKENTHAL partly grazed by Phenacovolva birostris (LINNÉ) and with egg masses laid by the latter. Tôsima, Sirahama, Wakayama Prefecture, September 1963 (collected by Mr. K. Sasaki and photo by Mr. T. TANAKA).

Fig. 9. A part of the same egg masses magnified. 

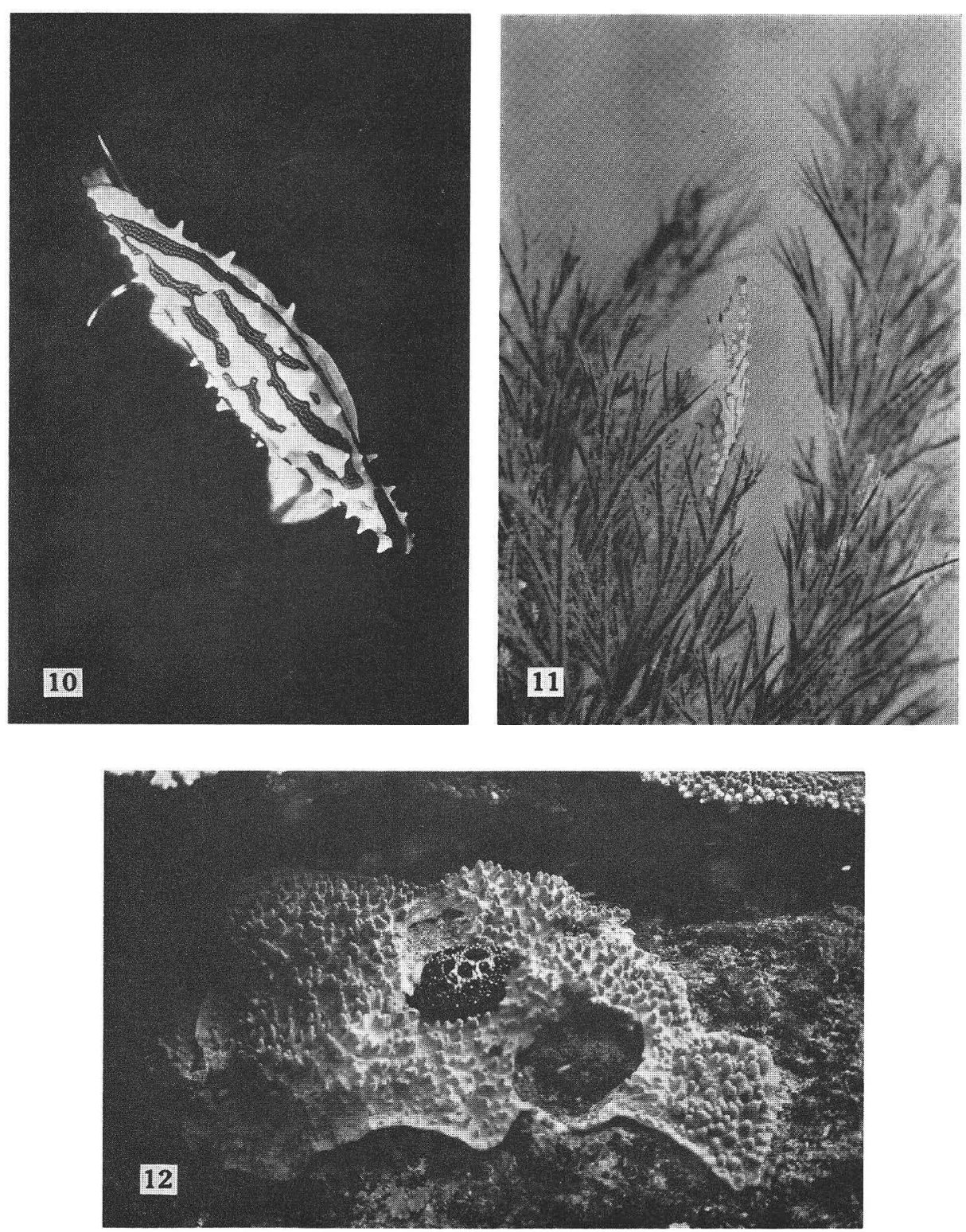

Fig. 10. Phenacovolva (Pellasimnia) subreflexa (AdAms \& ReEve), Tôsima, Sirahama, Wakayama Prefecture, November 1971 (collected and photo by Mr. Ch. ARAGA).

Fig. 11. The same animal on the gorgonid, Antipathes japonica Brook (photo by Mr. Ch. Araga). Fig. 12. Ovula ovum LinNé on Lobophyllum pauciforum (HeMrich \& EHRENBERG), Sabiura, Kusimoto, Wakayama Prefecture, December 1969 (collected and photo by Mr. S. FusE). 
1930 Diminovula verepunctata Iredale, Mem. Qeensl. Mus., 10, p. 85.

1932 Primovula (Diminovula) punctata, Schilder, Proc. Malac. Soc. London, 20, pp. 51, 58, pl. 3, fig. 5. 1962 Pseudosimnia (Diminovula) punctata, KIRA, Shells. West Pacific col., 1, p. 44, pl. 19, fig. 5.

Host and locality confirmed: Alcyonarian, Dendronephthya (Dendronephthya) nipponica Utinomi, (Seto, Sirahama, Wakayama Prefecture, July 1954; Seto, Sirahama, Wakayama Prefecture, April 1958; Sionomisaki, Wakayama Prefecture, May 1960; Sionomisaki, Wakayama Prefecture, May 1971)

Alcyonarian, Dendronephthya (Dendronephthya) gigantea (VERriLL), (Kusimoto, Wakayama Prefecture, April 1957; Sisôzima, Sirahama, Wakayama Prefecture, April 1958; Bansyozaki, Sirahama, Wakayama Prefecture, May 1958; Tôsima, Sirahama, Wakayama Prefecture, June 1958; Seto, Sirahama, Wakayama Prefecture, April 1959; Seto, Sirahama, Wakayama Prefecture, July 1959; Sionomisaki, Wakayama Prefecture, May 1960; Kusimoto, Wakayama Prefecture, March 1960; Seto, Sirahama, Wakayama Prefecture, April 1961; Takui, near Gobô, Wakayama Prefecture, February, 1961; Nada, Gobô, Wakayama Prefecture, February 1962; Sakai, Minabe, Wakayama Prefecture, December 1969)

Alcyonarian, Dendronephthya (Morchellana) habereri KüKENTHAL, (Sionomisaki, Wakayama Prefecture, May 1960, Sisôzima, Sirahama, Wakayama Prefecture, April 1961)

Alcyonarian, Dendronephthya (Morchellana) castanea UTinomi, (Nada, Gobô, Wakayama Prefecture, February 1962)

Alcyonarian, Stereonephthya rubriflora UTINomI, (Bansyozaki, Sirahama, Wakayama Prefecture, June 1956)

Alcyonarian, Stereonephthya japonica UTINomI, (Tôsima, Sirahama, Wakayama Prefecture, April 1959)

Distribution: Honsyû (northerly to Bôsô Peninsula), Sikoku, Kyûsyû, Also in the Indo-Pacific Region. (type locality-Bourbon, Reunion Island, Indian Ocean)

Remarks: This animal is symbiotic with Dendronephthya nipponica, laying eggs on the host and excreting faeces suffused with spicules of the host.

\section{Diminovula cristallina (KIENER)}

(Fig. 5)

1844 Ovula cristallina KIENER, Spec. Gen. Icon. Coquilles Vivants. Text 3, p. 14, pl. 4, fig. 3.

Host and locality confirmed: Alcyonarian, Stereonephthya japonica UTINomi, (Seto, Sirahama, Wakayama Prefecture, May 1954; Bansyozaki, Sirahama, Wakayama Prefecture, May 1958; Tôsima, Sirahama, Wakayama Prefecture, May 1960; Torinosu, Tanabe, Wakayama Prefecture, June 1969; Seto, Sirahama, Wakayama Prefecture. July 1970; Seto, Sirahama, Wakayama Prefecture, August 1971)

Alcyonarian, Stereonephthya rubriflora UTINomI, (Tôsima, Sirahama, Wakayama Prefecture, March 1959)

Distribution: Honsyû (northerly to Sagami Bay), Sikoku, Kyûsyû, China and 
also in the Indo-Pacific Region. (type locality-India)

\section{Diminovula florida (KURODA)}

1958 Pseudosimnia (Diminovula) florida Kuroda, Jap. Jour. Malac. Soc., 20 (2), pp. 172-173.

1963 Primovula (Diminovula) florida, Shikama \& Horikoshi, Serect. Shells World Illust. Colours. p. 44, pl. 32, fig. 17.

1971 Pseudosimnia (Diminovula) fulguris Azuma \& CATE, Veliger, 13 (3), pp. 267-268, fig. 15.

Host and locality confirmed: Alcyonarian, Alcyonium gracillimum KÜKENTHAL, (Tôsima, Sirahama, Wakayama Prefecture, November 1964; Ezura, Sirahama, Wakayama Prefecture, October 1970; Sakai, Minabe, Wakayama Prefecture, January 1972; Sakai, Minabe, Wakayama Prefecture, March 1972)

Distribution: Honsyû (northerly to Sagami Bay), (type locality-Sionomisaki, Wakayama Prefecture)

\section{Genus Phenacovolva IreDAlE}

\section{Phenacovolva birostris (LINNÉ)}

(Figs. 6-9)

1767 Bulla birostris, Syst. Nat., ed., 12, p. 1182.

1817 Radius brevirostris Schmacher, Essai Nouv. Syst. Vess. Test., p. 259.

1855 Volva rosea Adams \& Reeve, Proc. Soc. London, (1854), p. 130, pl. 28, fig. 9.

1865 Ovulum birostris, ReEve, Conch. Icon., Ovulum, sp. 45.

1932 Volva brevirostris, Schilder, Proc. Malac. Soc. London, 29, pp. 56, 62, pl. 5, figs. 74, 75.

1962 Volva (Phenacovolva) brevirostris rosea, Kira, Shells West. Pacific Col., 1, p. 45, pl. 19, figs. 10.

1963 Volva (Phenacovolva) brevirostris (Adams), Shikama \& Horikoshi, Select. Shells World Illust.

Colours, p. 45, pl. 32, fig. 27.

1963 Phenacovolva birostris, CERNoHorsky, Veliger, 10, p. 363, pl. 50, fig. 10.

Host and locality confirmed: Antipathid, Antipathes japonica BrooK, (Seto, Sirahama, Wakayama Prefecture, November 1957)

Gorgonid, Discogorgia sp., (Yuzaki, Sirahama, Wakayama Prefecture, April 1961)

Gorgonid, Anthiplexaura dimorpha (KüKenthal), (Tôsima, Sirahama, Wakayama Prefecture, May 1962; Tôsima, Sirahama, Wakayama Prefecture, June 1966; Tôsima, Sirahama, Wakayama Prefecture, August 1968, collected by K. SASAKI; Tôsima, Sirahama, Wakayama Prefecture, September 1970, collected by K. SASAKI)

Gorgonid, Acanthogorgia multispina KüKenthal \& GoRZALSKY, (Sakai, Minabe, Wakayama Prefecture, February 1971)

Gorgonid, Acalycigorgia inermis (HedLund), (Sakai, Minabe, Wakayama prefecture, January 1972)

Distribution: Honsyû (northerly to Bôsô Peninsula), Sikoku, Kyûsyû, also in the Western Pacific Region. (type locality-Ad Javam, Indonesia)

Remarks: This animal eats the polyps of the host (Anthoplexaura dimorpha) and exretes faeces suffused with spicules of the host. While breeding in the aquarium this animal laid eggs on the surface of the host. 


\section{Phenacovolva longirostris (SOWERBY)}

1828 Ovulum longirostris SoWERBY, Zool, Jour., 4 (14), p. 140.

1932 Volva birostris, Schilder, Proc. Malac. Soc. London, 20, pp. 56, 62, p1. 5, fig. 78.

1962 Volva (Phenacovolva) birostris. KIRA, Shells West. Pacific Col. 1, p. 45, pl. 19, fig. 11.

Host and locality confirmed: Gorgonid, Calicogorgia granulosa KüKenthal \& Gorzawsky, (Sakai, Minabe, Wakayama Prefecture, December 1969)

Distribution: Honsyû (northerly to Bôsô Peninsula), Sikoku, Kyûsyû. Also widely ranging in the Indo-Pacific Region. (type locality-Bourbon, Indian Ocean).

\section{Phenacovolva recurva (AdAMS \& REEVE)}

1848 Ovulum recurvum AdAMs \& ReEve, Zool. Samarang, Moll., p. 21, pl. 6, figs. 3a, b, c.

1931 Volva recurva, Schilder, Proc. Malac. Soc. London, 20 (1), pp. 56, 62.

Host and locality: Gorgonid, Antipathes japonica Brook, (Sakai, Minabe, Wakayama Prefecture, April 1971, collected by K. SASAKI).

Distribution: Honsyû (northerly to Sagami Bay), Sikoku, Kyûsyû, China. (type locality-China seas).

\section{Phenacovolva sowerbyana (WEINKAUFF)}

1758 Bulla spelta LINNÉ, Syst, Nat., p. 1182.

1865 Ovulum spelta, ReEve Conch. Icon., Ovulum sp. 42, pl. 10, figs. 42a, b.

1931 Volva sowerbyana, SchILdER, Proc. Malac. Soc. London. pp. 56, 62, pl. 5, fig. 73.

1964 Phenacovolva sowerbyana (WeinKaUfF), Habe, Shells West. Pacific col. 2, p. 64, pl. 19, fig. 12.

Host and locality confirmed: Gorgonid, Antipathes japonica BrooK, (Sakai, Minabe, Wakayama Prefecture, March 1970, collected by K. SASAKı).

Distribution: Honsyû (northerly to Ensyû-nada), Sikoku, Kyûsyû. Indo-Pacific Region. (type locality-Madagascar).

\section{Phenacovolva (Pellasimnia) subreflexa (AdAMs \& REEve)}

(Figs. 10-11)

1848 Ovulum subreflexa Adams \& ReEve, Zool. Samarang, Moll., p. 22, pl. 6, fig. 10.

1849 Ovulum philippinarum Sowerby, Proc. Zool. Soc. London, 16, p. 136.

1877 Volva carpenteri DUNKeR, Malak. Blatt., 24, p. 75.

1877 Volva adamsi DUNKer, Malak. Blatt., 24, p. 75. (non Ovulum adamsi Reeve, 1865)

1882 Radius carpenteri, DunKer, Index Moll. Mar. Japon., p. 102 pl. 13, figs. 1, 2.

1882 Radius adamsi Dunker, Index Moll. Mar. Japon., p. 102, pl. 13, figs. 3, 4.

1932 Volva philippinarum, Schilder, Proc. Malac. Soc. London, 20, pp. 56, 62, p1. 5, fig. 77.

1942 Neosimnia subrefrexa, YeN, Proc. Malac. Soc. London, 24, p. 212, pl. 17, fig. 101.

1962 Volva (Phenacovolva) philippinarum, KIRA, Shells West. Pacific col., 1, p. 45, pl. 19, fig. 9.

1968 Phenacovolva (Pellasimnia) philippinarum, CeRnohorsky, Veliger, 10, p. 364, pl. 31, fig. 12, 1971 Phenacovolva improcera Azuma \& Cate, Veliger, 13, p. 265, fig. 12.

Host and locality confirmed: Gorgonid, Melithaea flabellifera (KüKENTHAL), (Bansyozaki, Sirahama, Wakayama Prefecture, July 1959; Tôsima, Sirahama, Wakayama Prefecture, November 1963, collected by C. ArAGA). 
Distribution: Hosyû (northerly to Bôsô Peninsula), Sikoku, Kyûsyû. Widely ranging in the Tropical Pacific Region. (type locality-China).

\section{Genus Kuroshiovolva Azuma \& GATE}

\section{Kuroshiovolva shingoi AzUma \& CATE}

1971 Kuroshiovolva shigoi Azuma \& CATE, Veliger, 13 (3), pp. 266-267, figs. 14, 20, 21, 22, 23.

Host and locality confirmed: Gorgonid, Plumarella cristata KüKENTHAL \& GorZaWsky, (Sakai, Minabe, Wakayama Prefecture, April 1972, collected by K. SASAKI)

Distribution: Honsyû (type locality-off Hinomisaki, Kii Peninsula).

Genus Ovula BRUGUière

\section{Ovula ovum LINNÉ}

(Fig. 12)

1758 Bulla ovum LINNÉ, Syst. Nat., ed. 10, p. 725, no. 327.

1796 Volva cygnea RödING, Mus. Bolt., p. 21, no. 255.

1811 Ovula oviformis Lamarck., Ann. Mus. Hist. Nat., 16, p. 110.

1817 Ovula alba Schmacher., Essei Nouv. Syst., p. 258.

1828 Ovulum ovum var. pygmaea Sowerby, Zool. Jour. 4, p. 149.

1908 Amphiperas ovum (LinNé). Berch, Siboga Exp., 50, pl. 5, fig. 21 (animal).

Host and locality confirmed: Alcyonarian, Cladiella digitulata (KLunziger), (Ezura, Sirahama, Wakayama Prefecture, April 1957; Tôsima, Sirahama, Wakayama Prefecture, August 1972, collected by T. ÔsuKA).

Alcyonarian, Lobophytum pauciflorum (HEMrich \& EHRENBERG), (Sabiura, Kusimoto, Wakayama Prefecture, December 1969, collected by S. FUSE).

Alcyonarian, Lobophytum batarum Moser (owing to SIRAI's report).

Distribution: Honsyû (northerly to Kii Peninsula), Sikoku, Kyûsyû, Also wide ranging in the Tropical Indo-Pacific Region. (type locality-Amboina).

Remarks: While breeding in the aquarium the animal ate up Cladiella digitulata, (Diameter about $15 \mathrm{~cm}$.) in 12 days.

\section{Family Pediculariidae}

\section{Genus Pedicularia SwaInson}

26. Pedicularia (Pediculariona) pacifica PEASE

1863 Dentiora rubica PeAse, Proc. Zool. Soc. London, (1862), p. 240.

1865 Pedicularia pacifica PeASE, Proc. Zool. Soc. London, p. 516.

1903 Pedicularia stylastris Hedley, Mem. Austral. Mus., 4, p. 342, figs. 69, 70.

Host and locality confirmed: Hydrocoral, Stylaster profundiporus BROCH f. crassicaulis Broch, (Sakai, Minabe, Wakayama Prefecture, December 1968; Sakai, Minabe, Wakayama Prefecture, February 1970; Nada, Gobô, Wakayama Prefecture, April 
1971; Nada, Gobô, Wakayama Prefecture, May 1971; Sakai, Minabe, Wakayama Prefecture, March 1972)

Distribution: Honsyû (northerly to Bôsô Peninsula), Sikoku, Kyûsyû. Also wide ranging in the Tropical Pacific Region. (type locality-Gilbert Island)

\section{REFERENCES}

Azuma, M. \& C. N. Cate. 1971. Sixteen new species and one genus of Japanese Ovulidae. Veliger, 13 (3), 261-268, figs. $1-24$.

Baba, K. 1967. The picture of life in Primovula rhodia (Gastropoda). Collecting and Breeding, 29 (7), 233, figs. 2-4.

CERnohorsky, W. O. 1967. The Ovulidae, Pediculariidae and Triviidae of Fiji. Veliger, 10 (4), 353 374.

HABE, T. 1961. Coloured Illustrations of the Shells of Japan. 2. Hoikusha. Osaka. , 1964. Shells of the Western Pacific in Color. 2. Hoikusha. Osaka.

Habe, T. and T. Kuroda. 1965. A part of The New llustrated Encyclopedia of the Fauna of Japan. 2. Hokuryu-kan. Tokyo.

KIRA, T. 1962. Shells of the Western Pacific in Color. 2. Hoikusha. Osaka.

Kuroda, T. 1958. The Japanese species of Primovula series of the Amphiperatidae (Gastropoda). Venus, 20 (2), 167-173 (in Japanese)

Kuroda, T., Habe, T. and K. Oyama. 1971. The Sea Shells of Sagami Bay, 147-157, 96-103, pl. 24, figs. 1-33. Maruzen. Tokyo.

MASE, K. 1969. Amphiperatidae of Miura peninsula. Tiribotan, 5 (8), 219-221, pls. 8-9. (in Japanese).

Oyama, K. 1955. Pedicularia. Yumehamaguri, 81, 77, 78. (in Japanese).

SCHILDER, F. A. 1932. The living species of Amphiperatidae. Proc. Malac. Soc. London, 20, 46-64, pls. 3-5.

Shikama, T and M. Horikoshi. 1963. Selected Shells of the World illustrated in Colours. 44-46, pl. 32, figs. 10-32. Hokuryu-kan. Tokyo.

SHIRAI, S. 1967. The shells symbiotic with corals. 1. Collecting and Breeding, 29 (2), 58-61, figs. 1-5.

Yамамото, T. 1961. Ecology of primovula epibiotic on Octocorallia. Venus, 21 (2), 227-228. (in Japanese).

- 1963. Memoranda on animals and plants from Tanabe Bay, 2. Tanabe-Bunkazai, 7, 8092. (in Japanese).

, 1971. A new species of Primovula from Japan, parasitic to a gorgonid (Gastropoda:

Ovulidae). Publ. Seto Mar. Biol. Lab., 19 (4), 191-195, pl.8, figs. 1-4. 